\title{
FAD24, a Regulator of Adipogenesis, is Required for the Regulation of DNA Replication in Cell Proliferation
}

\author{
Yoshikazu Johmura, Shigehiro Osada, Makoto Nishizuka, and Masayoshi Imagawa* \\ Department of Molecular Biology, Graduate School of Pharmaceutical Sciences, Nagoya City University; 3-1 Tanabe- \\ dori, Mizuho-ku, Nagoya, Aichi 467-8603, Japan. \\ Received February 1, 2008; accepted March 3, 2008; published online March 10, 2008
}

\begin{abstract}
A novel gene, factor for adipocyte differentiation 24 (fad24), promotes adipogenesis by controlling DNA replication early on during a stage referred to as mitotic clonal expansion (MCE). MCE is considered distinct from the proliferation of pre-confluent cells, so we investigated the role of $f a d 24$ in the process. First, the expression of fad 24 was examined in pre-confluent and post-confluent 3T3-L1 preadipocytes, NIH-3T3 fibroblasts, and C2C12 myoblasts. $f a d 24$ was strongly expressed in the pre-confluent cells. The knockdown of $f a d 24$ by RNA interference impaired the ability of the pre-confluent cells to proliferate. Moreover, bromodeoxyuridine labeling and chromatin immunoprecipitation experiments indicated that the knockdown inhibited DNA synthesis by preventing the recruitment of histone acetyltransferase binding to ORC1 (HBO1), a component of the pre-replicative complex, to origins. fad 24 plays positive roles in the proliferation of pre-confluent cells as well as adipogenesis.
\end{abstract}

Key words DNA replication; cell proliferation; adipogenesis; fad24; histone acetyltransferase binding to ORC1 (HBO1)

DNA replication, one of the most fundamental steps of the cell division cycle, is strictly regulated by a complex network of extracellular and intracellular signaling pathways that control cell proliferation, differentiation, and apoptosis. To ensure proper genome duplication and inheritance, eukaryotic cells control the initiation of DNA replication through the precise assembly of the pre-replicative complex (pre-RC) at origins once in the same cell cycle. ${ }^{1,2)}$ The formation of the pre-RC, a prerequisite for the initiation of DNA replication, depends on the binding of the origin recognition complex (ORC) to origins. ${ }^{3)}$ ORC, in turn, recruits CDC6, CDT1, and eventually the minichromosome maintenance complex (MCM). ${ }^{4}$ 7) Recently, Iizuka et al. revealed that histone acetyltransferase binding to ORC1 (HBO1), a MYST family histone acetyltransferase (HAT), facilitates the recruitment of chromatin to the MCM necessary for the pre-RC's assembly. ${ }^{8)}$ However, the precise mechanism of replication initiation is still unclear, and some replication-initiation proteins may have not been discovered.

To elucidate the earliest step in adipocyte differentiation, we previously isolated 102 genes induced to express at the beginning of the differentiation of 3T3-L1 cells. ${ }^{9,10)}$ One of them was a novel gene, factor for adipocyte differentiation 24 (fad24). Gain-of-function and loss-of-function experiments indicated that fad 24 is a positive regulator of adipogenesis. ${ }^{11)}$ Moreover, our recent findings indicated that FAD24 interacts with components of Pre-RC, localizes to origins of DNA replication, and recruits HBO1, a component of pre$\mathrm{RC}$, to origins. Consequently, FAD24 promotes adipogenesis by controlling DNA replication early on during a stage referred to as mitotic clonal expansion (MCE). ${ }^{12)}$

After the induction of differentiation, post-confluent, growth-arrested 3T3-L1 preadipocytes synchronously reenter the cell cycle and undergo approximately two rounds of mitosis, known as MCE. ${ }^{13,14)}$ MCE appears to be necessary for adipocyte differentiation, and DNA replication and changes in chromatin structure during MCE might facilitate the transcription of genes leading to terminal differentiation. ${ }^{13,14)}$ MCE is considered different from the proliferation of preconfluent cells. In fact, some reports indicate that the tran- scription factor CCAAT/enhancer-binding protein (C/EBP) $\beta$ and $N$-acetyl-Leu-Leu-norleucinal (ALLN), a calpain inhibitor, play crucial roles in MCE but not in the proliferation of pre-confluent 3T3-L1 cells. ${ }^{15,16)}$ Therefore, studying whether FAD24 acts in concert with HBO1 to control DNA replication only in MCE during adipogenesis or in the proliferation of pre-confluent cells is very important for elucidating the function of this novel gene.

In this study, we show that $f a d 24$ is strongly expressed in pre-confluent 3T3-L1 preadipocytes, NIH-3T3 fibroblasts, and $\mathrm{C} 2 \mathrm{C} 12$ myoblasts as well as at the early stages of adipocyte differentiation. Cell proliferation was inhibited when the expression of $f a d 24$ was knocked down in these pre-confluent cells. Moreover, bromodeoxyuridine (BrdU) labeling and chromatin immunoprecipitation (ChIP) experiments indicated that FAD24 controls DNA replication in preconfluent cells through the recruitment of $\mathrm{HBO} 1$ to origins in the same way as MCE. These results imply that fad 24 plays positive roles in the proliferation of pre-confluent cells as well as adipogenesis.

\section{MATERIALS AND METHODS}

Cell Culture and Cell Counting Mouse 3T3-L1 preadipocyte cells, mouse NIH-3T3 fibroblastic cells, and mouse $\mathrm{C} 2 \mathrm{C} 12$ myoblasts were cultured as previously described. ${ }^{11)}$ For cell counting, cells were trypsinized and replated into 12 -well plates at a density of $1 \times 10^{4}$ cells/well. The cell numbers at different time points were measured with the WST-8 assay (TetraColor One, SEIKAGAKU CORPORATION) following the manufacturer's recommended protocols.

RNA Interference (RNAi) The shRNA expression plasmids for fad 24 and the control plasmid were used as described. ${ }^{11)}$ Transfection of the shRNA expression plasmids into 3T3-L1 and $\mathrm{C} 2 \mathrm{C} 12$ cells was performed as previously described, ${ }^{11)}$ except that the $\mathrm{C} 2 \mathrm{C} 12$ cells were transfected using program 'B-32' of Nucleofector (Amaxa).

RNA Isolation, Real-Time Quantitative PCR (Q-PCR), and Northern Blot Analyses Total RNA was extracted 
with TRIzol (GIBCO BRL Life Technologies) according to the manufacturer's instructions. The reverse transcription and Q-PCR were performed as previously described. ${ }^{11)}$ The predesigned primers and probe sets for fad 24 and 18S rRNA were obtained from Applied Biosystems. Northern blot analyses were also performed as described. ${ }^{11)}$

BrdU Labeling and Immunofluorescence For BrdU labeling and immunofluorescence microscopy, 3T3-L1 cells were plated on coverslips. At $24 \mathrm{~h}$ after plating, cells were labeled for $2 \mathrm{~h}$ with $30 \mu \mathrm{g} / \mathrm{ml}$ BrdU (BD Biosciences). Coverslips were fixed in $70 \%$ ethanol for $30 \mathrm{~min}$ and incubated in $100 \%$ methanol for $10 \mathrm{~min}$ at room temperature. The fixed cells on coverslips were then treated for $30 \mathrm{~min}$ with $1.5 \mathrm{M}$ $\mathrm{HCl}$, blocked with $0.5 \%$ Tween 20 in phosphate-buffered saline (PBS) for $5 \mathrm{~min}$, incubated with fluorescein isothiocyanate (FITC)-anti-BrdU monoclonal antibody (BD Biosciences) for $30 \mathrm{~min}$ at room temperature, and incubated with 7-amino-actinomycin D (BD Biosciences) for $5 \mathrm{~min}$ at room temperature. At each step described above, the cells were washed with PBS three times after treatment.

ChIP Assay Approximately $1.0 \times 10^{7}$ cells were incubated with $1 \%$ formaldehyde for $10 \mathrm{~min}$ at room temperature. Cross-linking was terminated by the addition of the stop solution (10 mm Tris- $\mathrm{HCl}, \mathrm{pH} 8.0$, and $2 \mathrm{~m}$ glycine). The cells were washed with PBS and then scraped into $1 \mathrm{ml}$ of the cold lysis buffer $(25 \mathrm{~mm}$ Tris-HCl, $\mathrm{pH} 7.5,1 \%$ Triton X-100, $0.1 \%$ SDS, $0.5 \%$ sodium deoxycholate, $5 \mathrm{~mm}$ EDTA, and $150 \mathrm{~mm} \mathrm{NaCl}$ ) containing the protease inhibitor mixture. These cell lysates were sonicated 10 times for $10 \mathrm{~s}$ each time to generate DNA fragments that ranged in size from 200 to $1000 \mathrm{bp}$. The sheared chromatin-lysed extracts were incubated with either $2 \mu \mathrm{g}$ of the anti-HBO1 antibody $(\mathrm{N}-18$, Santa Cruz Biotechnology) or $2 \mu \mathrm{g}$ of normal goat IgG (Santa Cruz Biotechnology) overnight at $4{ }^{\circ} \mathrm{C}$. These extractantibody mixtures were incubated for an additional $1 \mathrm{~h}$ with protein G-Sepharose beads (Amersham Bioscience). The immunoprecipitates were washed according to the conventional method, ${ }^{17}$ ) suspended in the extraction buffer (TE, $\mathrm{pH}$ 8.0, and $1 \%$ SDS), and incubated overnight at $65^{\circ} \mathrm{C}$ and for $2 \mathrm{~h}$ at $37^{\circ} \mathrm{C}$ with $100 \mu \mathrm{g}$ of proteinase $\mathrm{K}$ to reverse protein/DNA cross-links. Finally, these samples were processed for DNA purification by the phenol-chloroform extraction method and ethanol precipitation. PCRs were performed in $20 \mu \mathrm{l}$ with $1: 1000$ and $1: 2000$ of input DNA and $1: 10$ of the immunoprecipitates as described using the primers amplicon 15 and amplicon 19. ${ }^{18)}$ The number of PCR cycles yielding products within the linear range was determined using 2-fold serial dilutions of the input DNA. PCR products were separated on an $8 \%$ polyacrylamide gel and stained with ethidium bromide (EtBr). The intensities were determined with a fluoroimager (FluorImager 595, Amersham Biosciences).

\section{RESULTS}

Fad24 Is Strongly Expressed in Pre-confluent Cells as Well as at the Early Stages of Adipogenesis We recently showed that fad24 stimulates MCE and adipogenesis by positively regulating DNA replication through the recruitment of HBO 1 to origins, ${ }^{12}$ ) but it is unclear whether fad24 is required for DNA replication and cell proliferation in pre-confluent cells or not. First, we determined the expression of
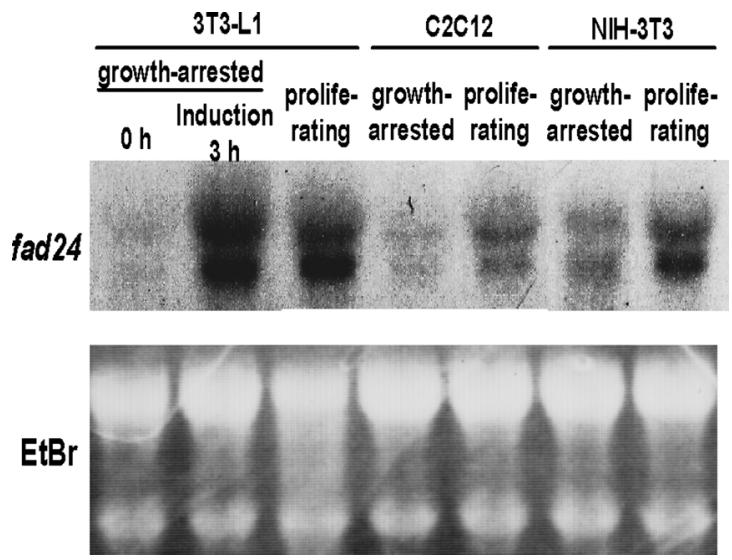

Fig. 1. Expression Profile of fad 24 in Pre-confluent and Post-confluent Cells

Total RNA was isolated from pre-confluent (proliferating) and post-confluen (growth-arrested) 3T3-L1, C2C12, and NIH-3T3 cells, or post-confluent 3T3-L1 cells at $3 \mathrm{~h}$ after adipogenic induction. Twenty-five micrograms of RNA was loaded in each lane and subjected to Northern blot analysis of fad24. Staining with EtBr for ribosomal RNA is shown as a loading control. This experiment was conducted twice and the reproducibility was confirmed.

fad24 in pre-confluent (proliferating) and post-confluent (growth-arrested) 3T3-L1 preadipocytes, C2C12 myoblasts, and NIH-3T3 cells by conducting Northern blots analyses. Fad24 was strongly expressed in the pre-confluent cells rather than post-confluent cells, although its expression was weak compared to that at $3 \mathrm{~h}$ after adipogenic induction (Fig. 1). These results indicate that the expression of fad 24 correlates with cell proliferation.

Fad24 Is Required for the Proliferation of Pre-confluent Cells As shown in Fig. 1, fad24 is thought to be involved in the proliferation of pre-confluent cells as well as MCE during adipocyte differentiation. Next, we examined the effect of knocking down fad 24 with RNAi on the proliferation of pre-confluent cells. The expression level of fad 24 in 3T3-L1 and C2C12 cells transfected with the shfad24 expression plasmid declined compared with that in control cells transfected with a scrambled shRNA expression plasmid (Fig. 2A). These cells were plated at a low density, and cell numbers were determined every $24 \mathrm{~h}$ by the WST- 8 assay. The numbers of shfad24-treated cells were lower than control values from 48 to $96 \mathrm{~h}$ after plating (Fig. 2B). These results indicate that $f a d 24$ is required for the proliferation of preconfluent cells as well as MCE.

The Knock Down of Fad24 Causes an Impairment of DNA Replication in Pre-confluent Cells Moreover, to explore the role of $f a d 24$ in cell proliferation, DNA synthesis was evaluated using BrdU labeling. Pre-confluent shfad24treated 3T3-L1 cells and control cells were pulse-labeled for $2 \mathrm{~h}$ with BrdU, and immunostained with the anti-BrdU antibody. The DNAs in the cells were also detected by 7-aminoactinomycin D. As shown in Fig. 3A, the incorporation of BrdU was detected in almost all control cells. On the other hand, less BrdU was incorporated into shfad24-treated cells than control cells, indicating that the knock down of fad 24 impaired DNA replication in pre-confluent cells (Figs. 3A, B).

The Knock Down of Fad24 Inhibits the Recruitment of HBO1 to Origins To limit replication to a single round per cell cycle, DNA replication can begin only when sufficient 


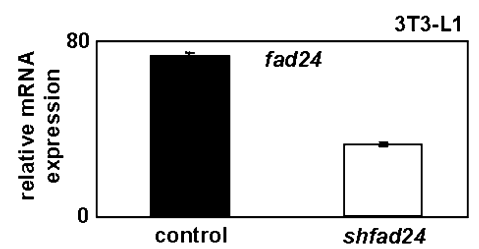

B

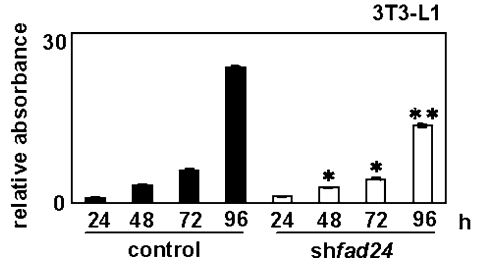

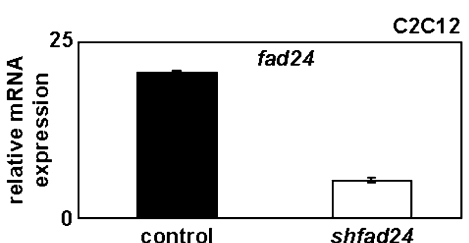

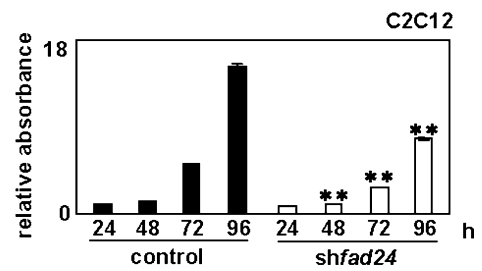

Fig. 2. Effect of the Knock Down of $f a d 24$ on the Proliferation of Pre-confluent Cells

(A) Q-PCR analyses of the expression of fad24. Total RNA was obtained from pre-confluent 3T3-L1 cells (left panel) and C2C12 cells (right panel) transfected with the plasmids expressing shRNA for fad24 (shfad24: white bar) or with plasmids expressing scrambled shRNA as a control (Control: black bar). The expression level of fad24 was determined by Q-PCR, and normalized with $18 \mathrm{~S}$ rRNA expression. (B) Effect of shRNA for fad 24 on the proliferation of pre-confluent 3T3-L1 and C2C12 cells. Shfad24-treated cells (white bars) or control cells (black bars) were plated at a low density. Cell numbers were determined at different time points after plating with the WST-8 assay. Each value represents the mean \pm standard deviation $(n=3)$. The asterisk and the double asterisks indicate significant differences when compared with the values of control cells at different time points $(p<0.05$ and $p<0.01$, respectively).

A

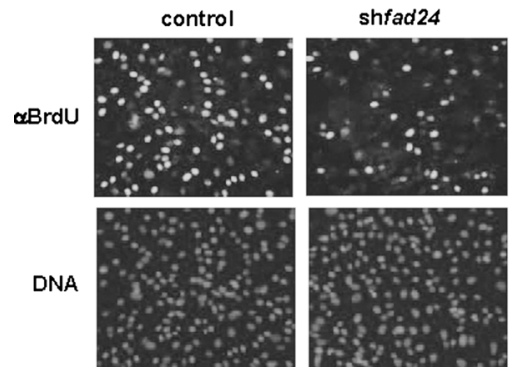

B

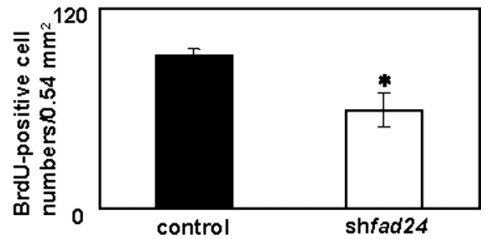

Fig. 3. The Inhibition of DNA Replication by the Knockdown of fad 24

(A) Effect of shRNA for fad24 on the incorporation of BrdU in pre-confluent 3T3-L1 cells. Pre-confluent shfad24-treated cells or control cells were labeled with BrdU, and stained with the FITC-conjugated anti-BrdU monoclonal antibody and 7-amino-actinomycin D (DNA). The fluorescence of BrdU (green) and DNA (red) was detected with a fluorescence microscope. (B) The cells labeled with BrdU in three microscopic view fields in (A) were counted for each experiment, and the results from three experiments were averaged. The value represents the mean \pm standard deviation $(n=3)$. The asterisk indicates significant difference when compared with the value for control cells $(p<0.05)$.

DNA replication initiation machinery is recruited to origins. $^{19)}$ As mentioned above, we revealed that fad24 positively regulates MCE through the recruitment of $\mathrm{HBO} 1$ to origins. ${ }^{12)}$ Then, we examined the effect on HBO1's localization to origins of fad24's knock down in pre-confluent cells. ChIP analyses were performed using pre-confluent shfad24treated 3T3-L1 cells and control cells. The immunoprecipitated DNA was amplified by PCR with primer pairs against a mouse origin (ORI), or an outside region (non-ORI) as a negative control. The signal against ORI was decreased in shfad24-treated cells compared with control cells (Figs. 4A, B). Consistent with these results, $\mathrm{fad} 24$ seems to be required for the recruitment of $\mathrm{HBO} 1$ to origins in pre-confluent cells.

\section{DISCUSSION}

In this study, we revealed that fad 24 is strongly expressed in pre-confluent cells as well as at the early stages in adipocyte differentiation. Our previous studies reported that the induction of $\mathrm{fad}_{24} \mathrm{expression}$ during adipogenesis is mainly dependent on insulin which is essential for MCE. ${ }^{12)}$ Therefore, the expression of $\mathrm{fad} 24$ seems to correlate with cell proliferation. Moreover, we revealed that human fad 24 is ubiquitously expressed in various tissues. ${ }^{11)}$ These results suggest that fad 24 is necessary for the proliferation of many types of cells.

It was revealed that the knock down of fad 24 expression inhibits the proliferation of pre-confluent cells as well as $\mathrm{MCE}$, an early and essential step in the differentiation program. It is clear that the cyclin-dependent kinase inhibitor p27 and the transcription factor $\mathrm{C} / \mathrm{EBP} \beta$ play crucial roles in MCE. ${ }^{15,16)}$ The inhibition of p27's degradation with ALLN and dominant-negative C/EBP blocked MCE, but not the proliferation of pre-confluent 3T3-L1 cells. ${ }^{15,16)}$ Considering that $\mathrm{C} / \mathrm{EBP} \beta$ is not expressed in pre-confluent $3 \mathrm{~T} 3-\mathrm{L} 1$ cells, ${ }^{20)}$ whether each gene product functions as a specific regulator for MCE might be determined mainly in the context of the expression pattern.

This study indicates that the knock down of fad 24 expression in pre-confluent 3T3-L1 cells impaired DNA replication by reducing the localization of HBO1 to origins in the same way as MCE. Nucleolar complex-associated protein 3 (Noc3p), a yeast ortholog of FAD24, plays a direct role in the initiation of DNA replication by interacting with MCM and ORC, as a cofactor for the recruitment of Cdc6p and MCM. ${ }^{21)}$ Moreover, Doyon et al. reported that HBO1 plays 
A

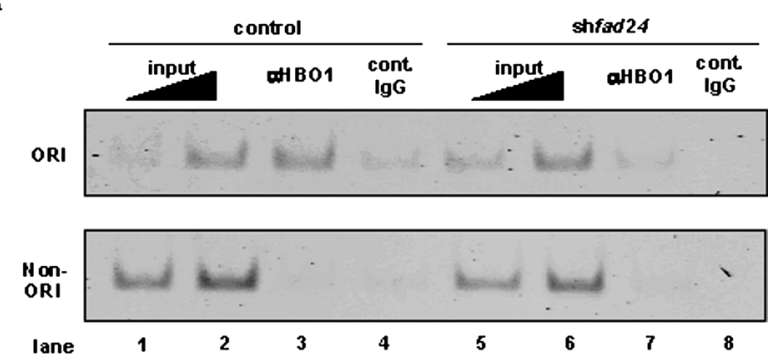

B

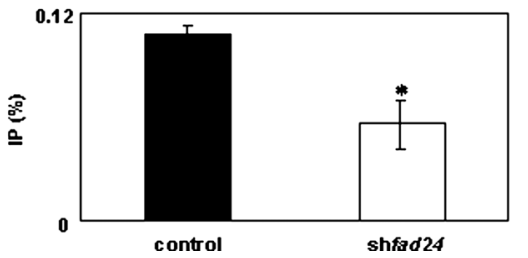

Fig. 4. Effect of the Knockdown of $f a d 24$ on the Localization of HBO1 to Origins of DNA Replication

(A) A ChIP assay was performed using pre-confluent 3T3-L1 cells transfected with the shfad24 expression plasmids or the control plasmids using the anti-HBO1 antibody (lanes 3,7 ), and normal goat IgG was used as a negative control (lanes 4,8 ). Input DNA $(1: 1000$ and $1: 2000$; lanes $1,2,5,6)$ and immunoprecipitated DNA were amplified by PCR with primer sets against the mouse DNA origins (ORI) or an outside region (non-ORI). PCR products were resolved on an $8 \%$ polyacrylamide gel and stained with EtBr. (B) The intensities in (A) were determined with a fluoroimager. This experiment was conducted three times, and ChIP efficiency against ORI was calculated as a percentage of immunoprecipitated material. Each value represents the mean \pm standard deviation $(n=3)$. The asterisk indicates a significant difference when compared with the value for control cells $(p<0.05)$.

essential roles in DNA replication in MCF7 and 293T cells. $^{22)}$ Therefore, FAD24 may act as a general cofactor for the recruitment of pre-RC including $\mathrm{HBO} 1$ to origins. At present, how FAD24 regulates pre-RC assembly and DNA replication with HBO1 remains largely unknown. HBO1 is the major histone $\mathrm{H} 4$ histone acetyltransferase, and chromatin structure modulated by histone modifications including acetylation has been linked to the control of pre-RC assembly and initiation of DNA replication in Saccharomyces cerevisiae, Xenopus, and Drosophila. ${ }^{23-25)}$ Consistent with these reports, FAD24 might act in concert with HBO1 to modulate chromatin structure around origins of DNA replication. In this article, we observed the effect of knocking down fad 24 on cell proliferation, but not the effect of overexpressing fad 24 . Recently, a growing body of data indicates that over-expression of individual pre-RC proteins results in uncontrolled cell cycle and tumorigenesis. ${ }^{19)}$ Therefore, it might be possible that over-expression of fad 24 in quiescent cells promotes cell proliferation. Further studies on the functions of $f a d 24$ in cell proliferation are definitely needed.

In summary, our findings indicate that fad 24 has important roles in cell proliferation as well as adipogenesis. It is possible that fad 24 is required for DNA replication and subsequent cell proliferation by recruiting $\mathrm{HBO} 1$ to origins in preconfluent cells. However, the precise regulation mechanisms of cell proliferation by fad 24 and also the contribution of fad24 to cell cycle are still unclear. Further analyses of the mechanism for the regulation of DNA replication by this novel gene are required and seem to provide insight into the molecular basis of replication initiation as well as adipogenesis. In addition, the abrogation of the exquisite control mechanisms that maintain the precise duplication of DNA replication results in detrimental gains and losses of genomic DNA commonly seen in cancer and developmental defects. ${ }^{19)}$ To elucidate the biological and biochemical roles of $\mathrm{fad} 24$ in these processes, the development and analyses of knockout mice and transgenic mice are ongoing.

Acknowledgements This work was supported in part by grants from the Ministry of Education, Culture, Sports, Science and Technology (MEXT), Japan, and Japan Society for the Promotion of Science (JSPS).

\section{REFERENCES}

1) Bell S. P., Dutta A., Annu. Rev. Biochem., 71, 333-374 (2002).

2) Blow J. J., Dutta A., Nat. Rev. Mol. Cell Biol., 6, 476-486 (2005).

3) Bell S. P., Stillman B., Nature (London), 357, 128-134 (1992).

4) Coleman T. R., Carpenter P. B., Dunphy W. G., Cell, 87, 53-63 (1996).

5) Donovan S., Harwood J., Drury L. S., Diffley J. F., Proc. Natl. Acad. Sci. U.S.A., 94, 5611-5616 (1997).

6) Maiorano D., Moreau J., Mechali M., Nature (London), 404, 622-625 (2000).

7) Nishitani H., Lygerou Z., Nishimoto T., Nurse P., Nature (London), 404, 625-628 (2000).

8) Iizuka M., Matsui T., Takisawa H., Smith M. M., Mol. Cell Biol., 26, 1098-1108 (2006).

9) Imagawa M., Tsuchiya T., Nishihara T., Biochem. Biophys. Res. Commun., 254, 299-305 (1999).

10) Nishizuka M., Tsuchiya T., Nishihara T., Imagawa M., Biochem. J., 361, 629-633 (2002).

11) Tominaga K., Johmura Y., Nishizuka M., Imagawa M., J. Cell Sci., 117, 6217-6226 (2004).

12) Johmura Y., Osada S., Nishizuka M., Imagawa M., J. Biol. Chem., 283, 2265-2274 (2008).

13) Hwang C., Loftus T., Mandrup S., Lane M. D., Annu. Rev. Cell Dev. Biol., 13, 231-259 (1997).

14) MacDougald O. A., Lane M. D., Annu. Rev. Biochem., 64, 345-373 (1995).

15) Zhang J.-W., Tang Q.-Q., Vinson C., Lane M. D., Proc. Natl. Acad. Sci. U.S.A., 101, $43-47$ (2004).

16) Patel Y. M., Lane M. D., J. Biol. Chem., 275, 17653-17660 (2000).

17) Ladenburger E. M., Keller C., Knippers R., Mol. Cell. Biol., 22, 1036-1048 (2002).

18) Rebecca K. R., Jeannie T. L., Mol. Cell. Biol., 26, 3707-3717 (2006).

19) Lau E., Tsuji T., Guo L., Lu S.-H., Jiang W., FASEB J., 14, 37863794 (2007).

20) Morrison R. F., Farmer S. R., J. Biol. Chem., 274, 17088-17097 (1999).

21) Zhang Y., Yu Z., Fu X., Liang C., Cell, 109, 849-860 (2002).

22) Doyon Y., Cayrou C., Ullah M., Landry A. J., Cote V., Selleck W., Lane W. S., Tan S., Yang X. J., Cote J., Mol. Cell, 21, 51-64 (2006).

23) Aggarwal B. D., Calvi B. R., Nature (London), 430, 372-376 (2004).

24) Danis E., Brodolin K., Menut S., Maiorano D., Girard-Reydet C., Mechali M., Nat. Cell Biol., 6, 721-730 (2004).

25) Pappas D. L., Jr., Frisch R., Weinreich M., Genes Dev., 18, 769-781 (2004). 